\title{
Evaluating Input Devices for Dance Research
}

\author{
Mari Romarheim Haugen and Kristian Nymoen \\ Department of Musicology, University of Oslo \\ \{m.r.haugen, kristian.nymoen\}@imv.uio.no
}

\begin{abstract}
Recording music-related motions in ecologically valid situations can be challenging. We investigate the performance of three devices providing 3D acceleration data, namely Axivity AX3, iPhone 4s and a Wii controller tracking rhythmic motions. The devices are benchmarked against an infrared motion capture system, tested on both simple and complex music-related body motions, and evaluations are presented of the data quality and and suitability for tracking music-related motions in real-world situations. The various systems represent different trade-offs with respect to data quality, user interface and physical attributes.
\end{abstract}

Keywords: Music and motion, dance, samba, motion capture, motion analysis, Qualisys, AX3, iPhone, Wii.

\section{Introduction}

A wide range of motion capture technologies enables measurements of musicrelated body motions. Infrared motion capture systems provide high precision position data and have successfully been used in a number of studies on musicrelated motions. However, these systems also have some limitations. If a marker gets occluded or moved outside the capture space, it will disappear from the recording, something that can restrict both the area for the performance, and also the number of people that can be captured simultaneously. In addition, the ecological validity of the experiment may be challenged by the visible reflective markers and the controlled environment required by the motion capture system. Previous studies suggest that using professional performers [9] or modifying the motion capture lab [13] can balance the influence of the artificial environment. Nevertheless, being able to record music-related motion in real-world situations, like a performing musician in a concert or a crowd dancing in a parade, is often desirable.

Several studies on music-related motion have used inertial sensors rather than optical systems. These are potentially less obtrusive and more affordable, thus possibly a better solution for studies where ecological validity is of importance. A popular choice among music researchers has been to use the Nintendo Wii controller $[1,3,15]$. Smartphones contain inertial sensors and have been deemed adequate for capturing the acceleration and orientation for certain types of motion [11]. Although smartphones have not been as popular as the Wii as recording device in studies on bodily motion, they have already been used extensively in musical interaction [14]. Besides the multi-purpose Wii and smartphones, other 
accelerometer-based sensing devices exist which are more dedicated to research purposes. The Axivity AX3 is one such device which has proven useful in recognizing everyday movement patterns [7]. It is clearly an advantage that these systems enable motion capture recordings outside a lab, but how do they perform in tracking of rhythmic motions in dance and other types of music-related motion? Our aim in this paper is to evaluate a selection of such devices with respect to the quality of the data they provided, but also their suitability for studies of music-related body motions in real-world situations.

\section{Devices}

The performances of three systems were evaluated in this study: The Axivity AX3, Apple iPhone and the Nintendo Wii controller. The AX3 is a logging device, meaning that data is recorded to the internal memory on the device without options for streaming the data to an external recording device. The iPhone and the Wii controller, on the other hand, both allow streaming of data. All systems provide three-dimensional acceleration data.

The dimensions of the Axivity AX $3^{1}$ device used in this study is $39 \times 36 \times 12.5$ $\mathrm{mm}$, with a mass of 19 grams (Fig. 1). The device has a built-in memory of $512 \mathrm{MB}$ to store the acceleration data and is charged and controlled over a USB connection to a computer. All control of the device is done using the PC software OM Gui v1.10 whilst being connected via USB. The recording starts immediately when the device is disconnected. The AX3 provides accelerometer data (including gravity) measured in Gs. The system was set to track at $100 \mathrm{~Hz}$.

An iPhone 4s, running iOS version 7.1.1 with dimensions 58.6x115.2x9.3 mm and with a mass of 138 grams (Fig. 1) was used. The iPhone data was sent via the application GyrOSC ${ }^{2}$ and recorded by a Max patch running on a MacBook Pro via Wi-Fi. The userAcceleration data (in Gs) is an estimate of the acceleration without gravity performed by the CMDeviceMotion class in Apple's API. The frame rate of the system was $40 \mathrm{~Hz}$.

We also used a Nintendo Wii controller with dimensions $36.2 \times 180 \times 30.8 \mathrm{~mm}$ and with a mass of 159 grams (Fig. 1). Accelerometer data from the Wii controller was received by OSCulator ${ }^{3}$ running on a MacBook Pro via Bluetooth and recorded using the software WiiDataCapture 2.1 [2]. The software imported the data to a text file containing time information and three-dimensional accelerometer data represented by numbers between 0 and 170 tracked at $100 \mathrm{~Hz}$.

For reference, high-precision position data was recorded using an optical motion capture system from Qualisys ${ }^{4}$. The system tracked the motion of a reflective marker (diameter $16 \mathrm{~mm}$ ) at a frame rate of $100 \mathrm{~Hz}$ (Fig. 1). Data was recorded into Qualisys Track Manager (QTM) 3.7. The position data from Qualisys is measured in millimetres $(\mathrm{mm})$.

\footnotetext{
${ }^{1}$ http://axivity.com

2 https://itunes .apple.com/us/app/gyrosc/id418751595?mt=8

${ }^{3}$ http://www.osculator.net

${ }^{4}$ http://qualisys.com
} 


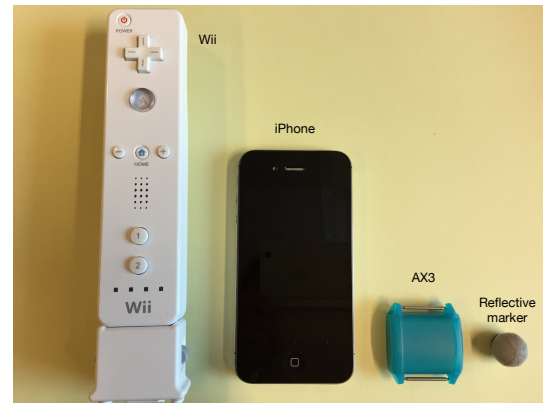

Fig. 1. The devices used in this study: Nintendo Wii controller, iPhone 4s and Axivity AX3, and also the reflective marker used in the Qualisys recording.

\section{Experiment}

A total of four recordings were carried out in the fourMs motion capture lab at the University of Oslo ${ }^{5}$. In order to record the same motions simultaneously in all devices, the AX3, iPhone, and Wii were strapped together and a reflective marker was attached. The basic noise level in the sensors was tested in the first recording with all devices lying stationary on the floor (Fig. 2 (left)). The second and third recording were designed to test how accurately the data from each of the devices reflected simple and complex motion patterns, respectively, to pre-recorded music. The music used in this study was a samba groove played on a traditional Brazilian hand drum called pandeiro. Samba music was chosen because of its systematic microtiming features and also because it has a corresponding dance involving complex body motion. This enables investigations of music-related motions of different complexities. The playback of the music was synchronized with the Qualisys recording. In the second recording simple motion resembling shaker motion was performed, holding the device in one hand (Fig. 2 (middle left)). In the third recording the devices tracked complex hip motions in samba dancing, with the devices attached on a dancer's hip (Fig. 2 (middle right)). In both recordings eight metronome clicks at 105 BPM were added to the audio track both before and after the samba groove. In the shaker recording impulsive motions, performed with the hand holding the devices, were carried out in synchrony with the metronome, and in the samba dance recording the person wearing the devices jumped in time with the metronome, creating acceleration peaks in all the data streams simultaneously. In both cases the resulting peaks in the data streams from all the devices were used as a common reference points when aligning the data. These synchronization motions were initially intended for aligning purposes only, however, they also proved to be a useful object of analysis. Such impulsive motions are indeed a type of musicrelated motion [5] and they provide yet another perspective on the differences between the recording devices.

In the first three recordings the acceleration data from the input devices was to be benchmarked and aligned with a Qualisys recording that in the shaker

\footnotetext{
${ }^{5}$ http://www .uio.no/english/research/groups/fourms/about/labs/
} 
and samba recordings was synchronized with the playback sound. Since we additionally were interested in investigating how input devices could be used in real-world situations, a fourth recording was carried out in order to test whether data from an input device could be synchronized with sound data without using an optical infrared system like Qualisys. The AX3 was chosen for this task due to its size and light-weight properties. Additionally, the AX3 does not require network access or connection to external hardware during the recording, something that can be useful in many situations. The AX3 tracked shaker motions performed to a samba groove and the sound was recorded using Logic Pro X software running on a Macintosh computer. The AX3 was attached to a wood block that was hit against another wood block in the beginning and in the end of the recording (Fig. 2 (right)). The resulting simultaneous spikes in the audio recording and the acceleration recording were used as common reference points when aligning the data streams.
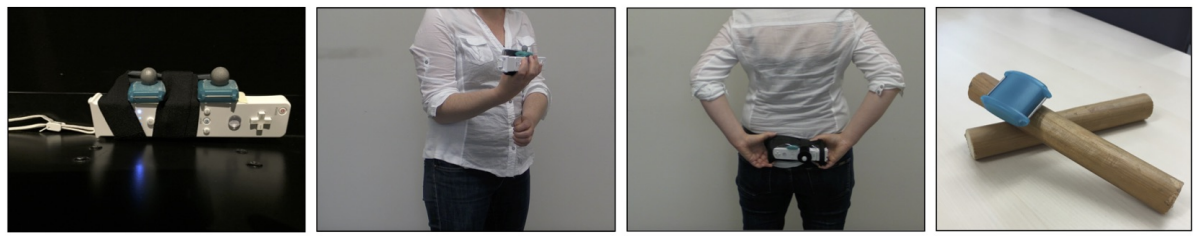

Fig. 2. Four recordings were carried out. (1) the devices lying staionary on the floor; (2) performing shaker motion to pre-recorded music while holding the devices in one hand; (3) dancing samba to pre-recorded music with the devices attatched to the dancer's hip; (4) a shaker recording using only an AX3 device and audio recording.

\section{Data processing}

The devices used in this study provide data in different units, and also differ in how they stream and store data. Consequently, some data processing was executed before the data could be analyzed.

\subsection{Post-processing}

Since the AX3 is a logging device, the data has to be downloaded from the device via USB and processed in the OM Gui software. We discovered a divergence between the specified sampling frequency of the AX3 and the actual sampling frequency. To investigate this two AX3 devices, both set to track at $100 \mathrm{~Hz}$, were used. Neither of the two devices did sample at the specified rate. The average frame rates of the two AX3 devices were found to be closer to $103 \mathrm{~Hz}$ and $95 \mathrm{~Hz}$, respectively. By exporting the AX3 data with time tags, that is, seconds relative to start instead of sample numbers, a more precise frame rate could be 
calculated. For this reason, AX3 data should be exported with time tags, rather than trusting the specified sampling rate.

The Qualisys position data was differentiated in order to compare it to the accelerometer-based devices. We applied a second order derivation with a Savitzky-Golay FIR smoothing filter (filter length of 5 frames). Alignment of individual axes of all devices is difficult, so our analysis in the following sections is based on the vector magnitude of the $3 \mathrm{D}$ acceleration data.

\subsection{Aligning the motion data}

The recorded data was imported into matlab. We implemented an automated process for aligning the data from the different devices. The data streams from the first three recordings were cropped and aligned to match the optical motion capture system. Each data stream was up-sampled to the frame rate of the Qualisys data using linear interpolation. Subsequently, cross-correlation was applied to calculate the time lag between the Qualisys data and each of the other data streams.

\section{$5 \quad$ Analysis and results}

The data was analyzed using the MoCap Toolbox [2] and custom made matlab scripts. The following sections present analysis and results from the four recordings: the noise estimation based on the recording of the devices lying still on the floor (section 5.1), impulsive motions to metronome and shaker motions to samba music (section 5.2), jumps to metronome and hip motions in samba dance to samba music (section 5.3), and a recording using only one accelerometer and sound recording (section 5.4).

\section{$5.1 \quad$ Noise}

The noise level in the devices was calculated as the standard deviations (SD) of the acceleration vector magnitude based on a 140 seconds long recording where the devices were lying stationary on the floor. In the beginning and in the end of the recording five impulsive motions were executed for alignment purposes. The recordings were subsequently cropped to the 140 seconds where the devices were lying still.

Since the AX3 and the iPhone provide data in gravity units, the acceleration data from QTM, measured in $\mathrm{mm} / \mathrm{s}^{2}$, was also converted to Gs. The unit of the Wii-recording is unknown, hence, in order to compare the noise level in the Wii with the other devices every data stream was normalized by the root-meansquare (RMS) values of the synchronization motions in the beginning and in the end of the recording. However, on closer look, it seems that the iPhone data does not show the highest peaks in the synchronization motion. Although not specified in the app used for acquiring iPhone data, various online discussion fora state that the iPhone acceleration data is limited to $\pm 2 \mathrm{G}$, which would 
explain why the iPhone data does not reflect the highest acceleration peaks. As a consequence of the limited range of the iPhone accelerometer the noise level of the iPhone is higher when normalized. Hence, the noise calculations based on both the raw data and the normalized data had to be included in the evaluation of the noise levels in the different devices.

The noise levels of the systems are displayed in Table 1. The first column displays the data as it is received directly from each system, with the exception of the QTM data which has been differentiated and converted to G unit. The second column shows the noise level normalized by the RMS value of the five synchronization motions. The results in Table 1 show quite low noise levels in Qualisys, iPhone and AX3. The data obtained from the Wii seems to be noisier than the data from the other systems. The jagged lines for the Wii in Fig. 3 shows that the bit depth of the data stream is lower than for the other devices (a range of less than 256 and resolution of 1 suggests 8 bits). This is possibly the reason why the Wii data is much noisier.

\begin{tabular}{l|ccc} 
& Raw data & Normalized (RMS) & \\
\cline { 1 - 2 } QTM & 0.80 & 0.92 & A QTM raw values converted to unit G. \\
AX3 & 2.64 & 1.88 & in the acceleration- calay filter was used \\
iPhone & 2.57 & 2.89 & cating comparisons between QTM andi- \\
Wii & 117.36 & 4.72 & the other devices.
\end{tabular}

Table 1. Noise in absolute acceleration data for 140 seconds of the stationary recording. The columns show the standard deviations (SD) of the raw acceleration data, and data normalized by the RMS of the synchronization motions. Values are in $10^{-3}$ unit.

\subsection{Impulsive motions to metronome and shaker motions to samba music}

The second recording was done to investigate how well the devices track impulsive and simple shaker motions. All the devices were held in one hand while performing shaker motions in accordance with the music (Fig. 2 (middle)). Impulsive synchronization motions were executed in the beginning and in the end of the recording. Since the unit of the Wii-recording is unknown, every data stream was normalized by the root-mean-square (RMS) value of the entire recording. Fig. 3 shows the entire shaker recording (top), and a five-second excerpt (bottom). All devices follow the general tendencies recorded by the Qualisys system in this recording. The low bit depth of the Wii indicated by the jagged purple line was compensated for by applying a Butterworth smoothing filter.

When tracking the synchronization motion, the data streams from QTM, AX3 and Wii all showed clear spikes that seem to be in accordance with the metronome (Fig. 4). The data stream from the iPhone also showed acceleration peaks, however, limited by the range of the accelerometer as previously discussed. 


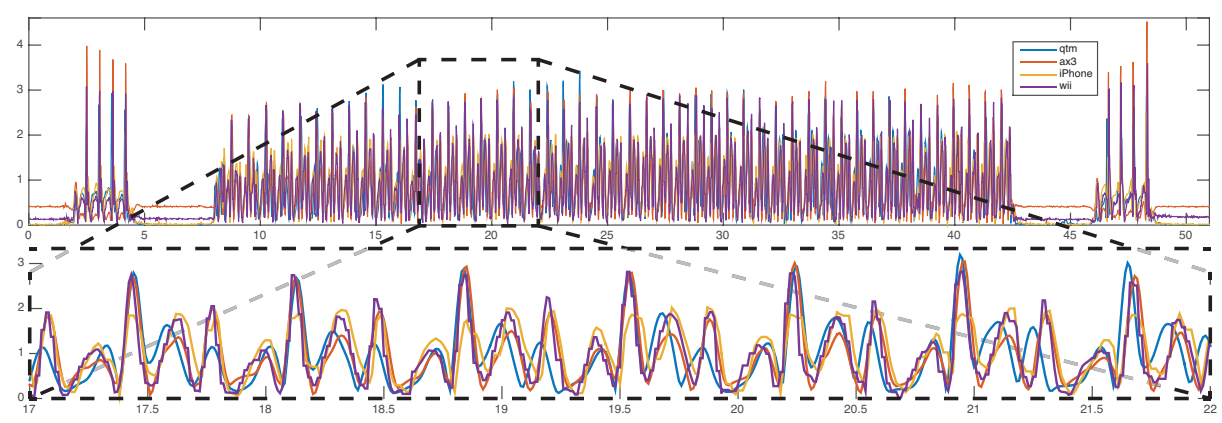

Fig. 3. Visual comparison between data from the different devices in the shaker recording (after RMS normalisation).
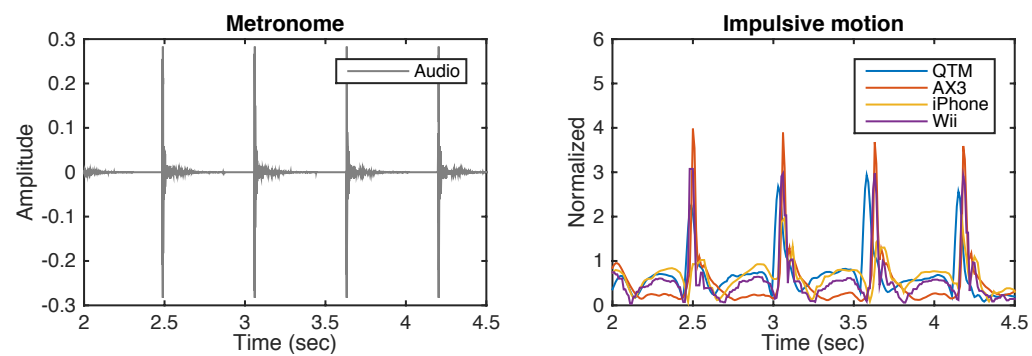

Fig. 4. The correspondence between metronome (left) and the impulsive motion holding the devices in one hand (right). 
Next, we investigated the correspondence between the shaker motion and a samba groove. A comparison between the acceleration plots and the plotted audio waveform reveals that there are four acceleration peaks in each beat, which indicates that the recorded shaker motion may correspond with the 16th-note level in the music (Fig. 5). According to previous research it has been suggested that the 4th 16th-note in a beat plays a significant role in samba groove being both longer in duration $[4,6,10]$ and also more accentuated than the others [4]. A qualitative evaluation of the fluctuation in acceleration amplitude in the shaker motion showed that both QTM, AX3 and Wii indicated a higher acceleration peak at the 4 th peak in each measure than the others. The acceleration peaks in the iPhone data stream, on the other hand, showed approximately the same strength, something that supports the previous suggestion that the iPhone is unable to pick up the highest acceleration peaks (see Noise section in 5.1).
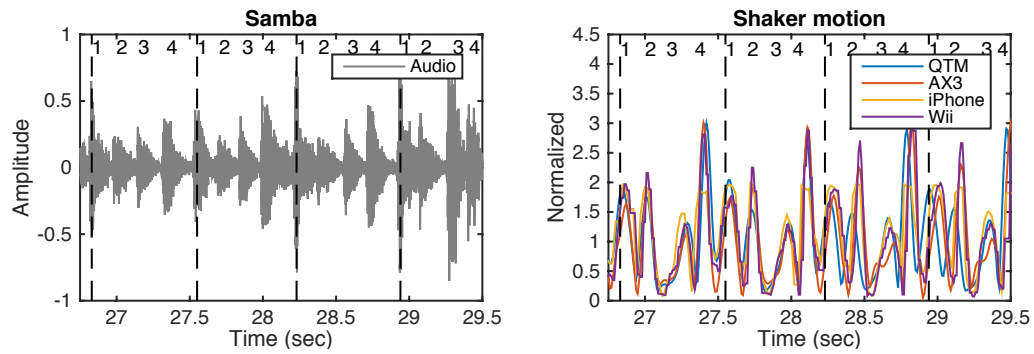

Fig. 5. The correspondence between samba music (left) and shaker motion (right) in two measures. The beat positions are indicated by dashed lines and 16th-note positions represented as numbers between $1-4$. The shaker motion seems to correspond with the 16th-note level.

In order to investigate how accurately the acceleration peaks reflect the duration pattern on the 16th-note level we calculated the 16th-note duration in the music and the duration between corresponding acceleration peaks in the shaker motion in a total of four measures. Since all the 16th-notes were present in the sound as sudden increase in energy, their temporal position could be identified using the onset detection function in the MIRtoolbox for matlab [8]. The 16thnote durations were subsequently calculated by measuring the time from the onset of one 16th-note to the next. The temporal position of the acceleration peaks were estimated using a custom made matlab function picking peaks in a graph based on directional changes. The duration pattern based on the motion data was calculated by measuring the time of one peak to the next. Our audio analysis revealed a medium - medium - medium - long duration pattern on 16th-note level. However, our motion analysis showed that the person performing the shaker motions seemed to perform a motion duration pattern deviating from the audio 16th-note duration pattern. The QTM data showed that the shaker motion in this recording follows a medium - long - short - medium duration pattern. This duration pattern was also recognized by the AX3 and Wii. The iPhone data, on the other hand, showed a medium - medium - long - 
medium pattern. The low clipping threshold on the iPhone accelerometer data makes the peak times more difficult to detect. This may be the reason for the deviating duration pattern in the iPhone data.

\subsection{Jumps to metronome and hip motions in samba dance}

A more complicated task than tracking shaker motion is the recording of hip motions in samba dance. In the third recording the devices were strapped onto the dancer's hip. Before and after the samba groove the dancer jumped in synchrony with the metronome.

The acceleration peaks caused by the synchronization jumping was recognizable in the QTM, AX3 and Wii, however, followed by a second lower peak. This is probably due to flexible knees allowing the body to bounce after the jump. In addition, jump preparation motions are recognized ahead of the peaks. Again, the iPhone was unable to pick up the highest acceleration peaks. A visualization of the metronome and the corresponding jumps is presented in Fig 6 .
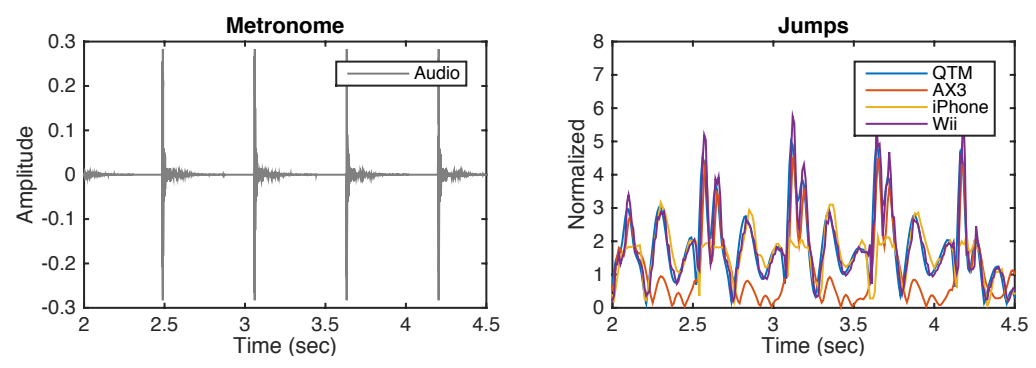

Fig. 6. The correspondence between metronome (left) and jumps (right). In addition to the peaks related to the temporal points when the dancer's feet meet the floor after the jump, preparation motions before the jump and bouncing motions after the jump is tracked.

The hip motion in samba dance are complex and involves a number of rapid direction changes. The motion pattern is periodic, however, a rhythm pattern is not easily detected by looking at the acceleration plots. In the recording of the simple shaker motion to samba music, the motion curves from AX3 and Wii were very similar to the QTM curve (see Fig. 5). In the hip recordings, on the other hand, the resemblance between the QTM data and the data from the other devices is less clear. A qualitative evaluation of the fluctuation in acceleration amplitude do however indicate a slight increase in acceleration around the 2nd and 4th 16-note in all devices. A visualization of the samba music and the corresponding dance is presented in Fig 7. 

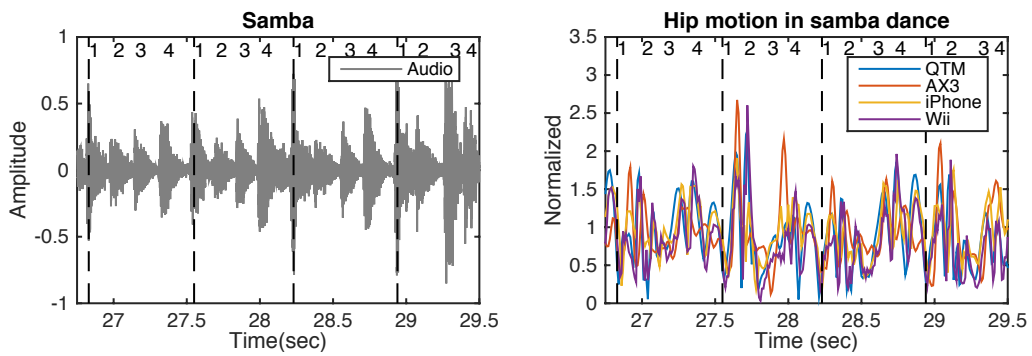

Fig. 7. The correspondence between samba music (left) and hip motion in samba dance (right) in two measures. The beat positions are indicated by dashed lines and 16th-note positions represented as numbers between $1-4$.

\subsection{Sound and motion recording.}

In the recordings presented above, the data from the portable devices were synchronized using cross-correlation against data from a state-of-the-art motion capture system. However, our motivation for testing the smaller devices in the first place is the use of such equipment outside a lab. In research on music and motion it is important to be able to do synchronized recordings of sound and motion. Accordingly, we did a recording where the AX3 tracked shaker motions performed to a samba groove and simultaneous audio recording using Logic Pro.

As described earlier the AX3 was attached to a wood block that was hit against another wood block creating simultaneous spikes in the beginning and end of both the sound and acceleration recordings (see Experiment section 3). The data streams were aligned by first transforming the audio recording into an envelope representation. Exerpts containing the synchronization motion and sound were extracted from both the sound and motion recording and crosscorrelation was used to determine the time difference between the two.

Plots of the aligned sound and motion data is displayed in Fig. 8. The automated synchronization process appears to have aligned the two data streams satisfactorily. This suggests that recordings of music-related motion can be carried out in real-world situations using only an AX3 device and a sound recorder, as long as synchronization actions are carried out in the beginning and in the end of the experiment.

\section{Discussion}

In this paper we have evaluated the performance of three input devices, namely AX3, iPhone and Wii, tracking music-related motions featured by different qualities. The devices were benchmarked against an optical infrared motion capture system from Qualisys.

In order to compare data streams residual analysis or correlation might be suggested. However, with residual analysis, the effects of minor clock drift in each system would be drastic, even if the magnitude of the acceleration measurement 

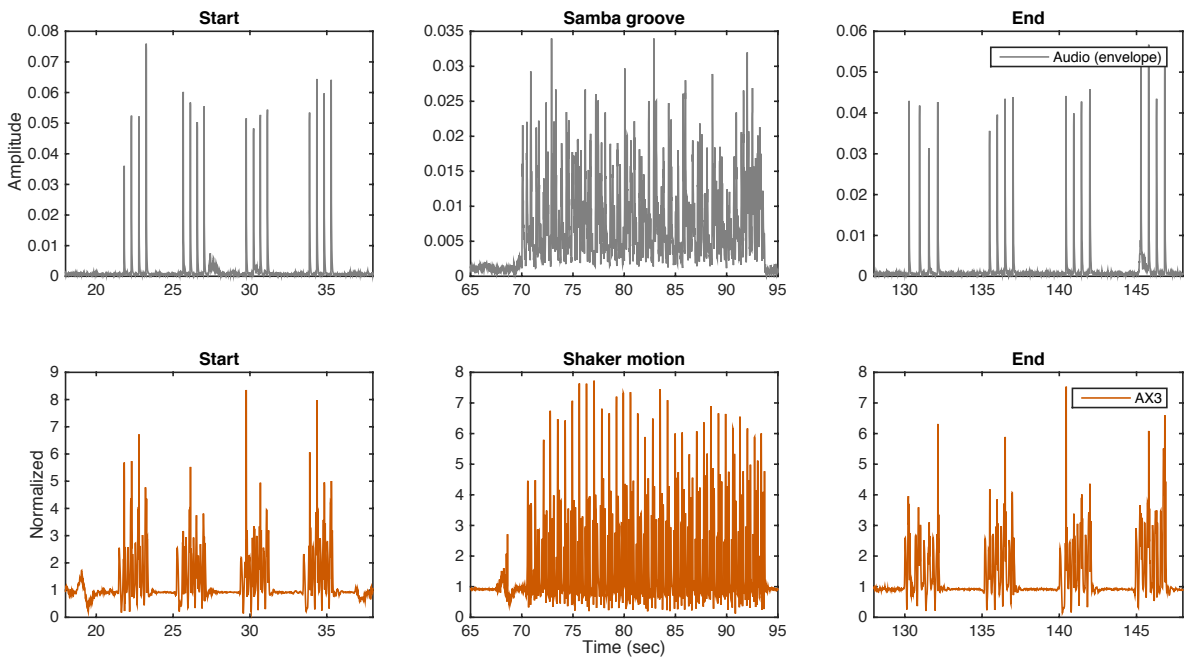

Fig. 8. Plot showing the audio envelope from a sound recording and data from the AX3 recording. The sections that are containing the synchronized peaks and spikes at the beginning (left) and in the end (right), and also the samba groove with corresponding shaker motion (middle) are extracted.

is precise. Correlation analysis is also problematic due to autocorrelation [12]. For this reason, initial comparison between the data streams was done by visual inspection of the data streams from each device to the same recording.

Synchronization of all the devices was possible using the data obtained from the tracking of impulsive motions and jumps to a metronome in the beginning and in the end of each recording. In the jump recording the acceleration peaks was surrounded by multiple peaks caused by jump preparation motions and flexible knees after the landing. The participant in this study was instructed to land the jump in synchrony with the metronome. Jumping in synchrony with a metronome could, however, have a number of different interpretations. So in recordings where a device is to be attached onto a person, it would be better to perform synchronization actions similar to our impulsive hand motion before the the sensor is attached to the person to ensure unambiguous acceleration spikes.

In the recording of impulsive hand motion and jump, the AX3 and the Wii both showed clear acceleration spikes. The iPhone data did also show acceleration spikes, however, not as clear as the others. This is probably because the iPhone data did not fully reflect the magnitude of the acceleration due to limited range of the accelerometer. This means that if motions resulting in high acceleration values are to be recorded and analyzed, an iPhone should not be used.

In the analysis of the simple shaker motion to samba music, we found that all the devices seemed to capture the general tendency. In order to investigate 
the precision of the acceleration peaks we calculated the inter onset intervals (IOIs) between the peaks. The motion analysis based on the QTM data revealed a medium - long - short - medium duration pattern on 16th-note level, a duration pattern that was recognized by AX3 and Wii as well. Again, the precision of the peaks in the data stream from the iPhone was low, resulting in a diverging duration pattern. This suggests that data obtained from AX3 and Wii can be used for detecting rhythmical patterns in simple motion based on acceleration peaks, while the data obtained from iPhone is too imprecise.

When tracking complex hip motions in samba dance, none of the data streams seemed to follow the QTM recording accurately. The hip motions in samba dance is featured by consecutive rapid directional changes, something that seem to be a challenge for the input devices. The data may not be precise enough to capture complex motion with rapid acceleration fluctuations. The iPhone may also be restrained by its low frequency rate $(40 \mathrm{~Hz})$. A qualitative examination of the fluctuation in acceleration amplitude does however indicate a slight increase in acceleration around the corresponding 2 nd and the 4th 16-note in the music.

We also tested whether data from an input device could be aligned with a audio recording without using an optical infrared system. We found that data from AX3 could be aligned with the audio recording alone, understood that impulsive sound-producing actions, producing unambiguous spikes in the acceleration data and peaks in the sound recording simultaneously is carried out both in the beginning and in the end of each recording. This opens for a lot of possibilities since this suggests that music-related motions can be captured in ecologically valid environments using only an audio recorder and an input device.

In dance research, and also when recording body motions in musical performances, the size and the weight of the sensors being used must be considered. Because the AX3 is so small it can easily be attached to a participant's body, or even be carried in a pocket, without interfering with the music performance or experience. The built in memory chip also allows for long recordings. However, a disadvantage is the divergence in sample rate between devices. This can however be sidestepped by exporting the AX3 data with time tags instead of sample numbers.

\section{Conclusion}

Recording music-related motions in an ecologically valid environment can be challenging. Although optical infrared motion capture systems are unrivaled when it comes to data quality, there are some challenges regarding marker occlusion, limited capture space and ecological validity. In this study we have evaluated the performance of three sensing systems, namely AX3, iPhone and Wii and compared it to an infrared motion capture system from Qualisys. None of the systems could provide as precise data as an optical infrared motion capture system, however, the data obtained from AX3 and Wii was proven to be useful for analyzing rhythmical structures in music-related motions. In addition, the small and light-weight feature of the AX3 device makes it preferable in many 
situations. The various systems evaluated in this study represent different trade offs with respect to data quality, user interface and physical attributes.

\section{References}

1. Amelynck, D., Grachten, M., Van Noorden, L., Leman, M.: Toward e-motion-based music retrieval a study of affective gesture recognition. Affective Computing, IEEE Transactions on 3(2), $250-259$ (2012)

2. Burger, B., Toiviainen, P.: Mocap toolbox - A matlab toolbox for computational analysis of movement data. In: Bresin, R. (ed.) Proceedings of the 10th Sound and Music Computing Conference, (SMC). pp. 172 - 178 (2013)

3. De Bruyn, L., Leman, M., Moelants, D., Demey, M.: Does social interaction activate music listeners? In: Computer Music Modeling and Retrieval. Genesis of Meaning in Sound and Music, pp. 93 - 106. Springer (2009)

4. Gerischer, C.: O suingue baiano: Rhythmic feeling and microrhythmic phenomena in brazilian percussion. Ethnomusicology 50(1), 99 - 119 (2006)

5. Godøy, R.I.: Gestural-sonorous objects: embodied extensions of schaeffer's conceptual apparatus. Organised Sound 11(02), 149 - 157 (2006)

6. Gouyon, F.: Microtiming in samba de roda - Preliminary experiments with polyphonic audio. In: SBCM 2007 Proceedings. pp. 197 - 203 (2007)

7. Khan, A.M.: Recognizing physical activities using the axivity device. In: The Fifth International Conference on eHealth, Telemedicine, and Social Medicine (2013)

8. Lartillot, O., Toiviainen, P.: A matlab toolbox for musical feature extraction from audio. In: International Conference on Digital Audio Effects (2007)

9. Naveda, L., Leman, M.: Sonification of samba dance using periodic pattern analysis. In: ARTECH 2008. 4th International Conference on Digital Arts. pp. $16-26$ (2008)

10. Naveda, L., Leman, M.: A cross-modal heuristic for periodic pattern analysis of samba music and dance. Journal of New Music Reseach 38(3), 255 - 283 (2009)

11. Nymoen, K., Volsund, A., Skogstad, S.A., Jensenius, A.R., Torresen, J.: Comparing motion data from an ipod touch to an optical infrared marker-based motion capture system. In: Proc. International Conference on New Interfaces for Musical Expression. pp. 88 - 91 (2012)

12. Schubert, E.: Correlation analysis of continuous emotional response to music: Correcting for the effects of serial correlation. Musicae scientiae 5(1 suppl), $213-236$ (2002)

13. Van Dyck, E., Moelants, D., Demey, M., Deweppe, A., Coussement, P., Leman, M.: The impact of the bass drum on human dance movement. Music Perception: An Interdisciplinary Journal 30(4), 349 - 359 (2013)

14. Wang, G.: Designing smule's ocarina: The iphone's magic flute. In: Proceedings of the International Conference on New Interfaces for Musical Expression. pp. $303-$ 307. Pittsburgh, PA (2009)

15. Witek, M.: '... and I feel good!' : the relationship between body-movement, pleasure and groove in music. Ph.D. thesis, University of Oxford (2013) 